\title{
High levels of X-linked Inhibitor-of-Apoptosis Protein (XIAP) are indicative of radio chemotherapy resistance in rectal cancer
}

\author{
L. Flanagan ${ }^{1,2}$, J. Kehoe ${ }^{1,3,4}$, J. Fay ${ }^{3}$, O. Bacon ${ }^{1}$, A.U. Lindner ${ }^{1,2}$, E.W. Kay ${ }^{3}$, J. Deasy ${ }^{4}$, D.A. McNamara ${ }^{4}$ \\ and J.H.M. Prehn ${ }^{1,2^{*}}$
}

\begin{abstract}
Background: The mainstay of treatment in rectal cancer is neoadjuvant radio chemotherapy prior to surgery, in an attempt to downstage the tumour, allowing for more complete removal during surgery. In $40 \%$ of cases however, this neoadjuvant radio chemotherapy fails to achieve tumour regression, partly due insufficient apoptosis signaling. X-linked Inhibitor of Apoptosis Protein (XIAP) is an anti-apoptotic protein that has been reported to contribute to disease progression and chemotherapy resistance.
\end{abstract}

Methods: We obtained rectal biopsy normal and matched tumour tissue from 29 rectal cancer patients with varying degrees of tumour regression, and using Western blot, examined anti-apoptotic XIAP and pro-apoptotic Smac protein levels in these tissues, with the aim to examine whether disturbed XIAP/Smac levels may be an indicator of neoadjuvant radio chemotherapy resistance. Expression of inhibitor of apoptosis proteins CIAP-1 and CIAP-2 was also examined.

Results: We found that levels of XIAP increased in accordance with the degree of radio chemotherapy resistance of the tissue. Levels of this protein were also significantly higher in tumour tissue, compared to matched normal tissue in highly resistant tissue. In contrast, Smac protein levels did not increase with radio chemotherapy resistance, and the protein was similarly expressed in normal and tumour tissue, indicating a shift in the balance of these proteins. Post treatment surgical resection tissue was available for 8 patients. When we compared matched tissue pre- and post- radio chemotherapy we found that XIAP levels increased significantly during treatment in both normal and tumour tissue, while Smac levels did not change. CIAP-1 and CIAP-2 levels were not differentially expressed in varying degrees of radio chemotherapy resistance, and neoadjuvant therapy did not alter expression of these proteins.

Conclusion: These data indicate that disturbance of the XIAP/Smac balance may be a driver of radio chemotherapy resistance, and hence high levels of XIAP may be a useful indicator of neoadjuvant radio chemotherapy resistance in rectal cancer. Moreover, as XIAP levels increase with radio chemotherapy it is possible that a subset of more resistant tumour cells survive this treatment and may be resistant to further adjuvant treatment. Patients with resistant tumours highly expressing XIAP may benefit from alternative treatment strategies, such as Smac mimetics post neoadjuvant radio chemotherapy.

Keywords: Rectal cancer, Neoadjuvant radio chemotherapy, Apoptosis, Inhibitor of apoptosis proteins

\footnotetext{
* Correspondence: prehn@rcsi.ie

${ }^{1}$ Department of Physiology and Medical Physics, Royal College of Surgeons

in Ireland, 123 St. Stephen's Green, Dublin 2, Ireland

${ }^{2}$ Centre for Systems Medicine, Royal College of Surgeons in Ireland, 123 St.

Stephen's Green, Dublin 2, Ireland

Full list of author information is available at the end of the article
} 


\section{Background}

Under homeostatic conditions a balance is struck between cell survival and cell death. Dysregulation of proteins involved in apoptosis, or programmed cell death, can upset this balance and push cells towards a disease state. Excessive apoptosis can lead to neurodegenerative diseases, and conversely situations where apoptosis is deficient can result in carcinogenesis and chemo resistance [1-4]. In the rectal cancer treatment setting neoadjuvant radio chemotherapy is performed prior to surgery in the hope of downsizing the tumour, allowing for more complete removal during surgery [5]. Responses to neoadjuvant radio chemotherapy can vary greatly between patients, and are categorised according to the Royal College of Pathologists depending on extent of tumour regression as RCPath $\mathrm{A}$ (complete tumour regression), RCPath B (partial tumour regression), or RCPath C (no marked tumour regression) [6]. In 15-27\% of patients therapy is successful and a complete pathological response is achieved, with patients displaying no residual tumour [7-9]. On the other hand, in $30-40 \%$ of patients no tumour regression is seen following neoadjuvant radio chemotherapy $[9,10]$. Molecular biomarkers indicative of treatment response could help streamline treatment selection and spare some patients from undergoing inefficient treatments.

Defective or deregulated apoptosis may be the driving force behind this chemo resistance. X-linked Inhibitor of Apoptosis Protein (XIAP) is an anti-apoptotic protein that acts to suppress cell death via potent inhibition of caspases [11-13]. High expression of this protein corresponds to poor prognosis in many cancers [14-16], and furthermore its expression level correlates positively with disease progression [17]. As well as contributing to disease progression, XIAP was also reported to contribute to chemotherapy resistance, and targeting this protein was found to effectively sensitise cells to apoptosis and suppress tumour progression [18-21]. Second mitochondriaderived activator of caspases (Smac) is a potent inhibitor of XIAP. By binding to XIAP, Smac relieves caspase inhibition and allows apoptosis to proceed. Cytosolic Smac also binds to cIAP-1 and-2, inducing rapid autoubiquitination and proteasomal degradation of Smac and cIAPs, resulting in an NF-KB-dependent secretion of TNF- $\alpha$ and subsequent autocrine apoptosis induction [22-24]. In cancer cells where XIAP is over expressed Smac levels may be insufficient to block XIAP activity, and this disturbed XIAP/Smac balance may contribute to apoptotic resistance. Studies demonstrate that restoration of the XIAP/Smac balance in chemotherapy resistant cancer cells can re-establish apoptosis $[25,26]$. As a result synthetic Smac peptides are being investigated for their therapeutic value in cancer. In conjunction with other drugs, such as TRAIL, these compounds have been successful in sensitising cancer cells to apoptosis and are currently in clinical trials [27].

A better understanding of the molecular changes occurring during neoadjuvant radio chemotherapy could help identify markers of response, or tools to monitor treatment response. Here we performed a quantitative analysis of XIAP and Smac protein levels in the pretreatment biopsy tissue of a cohort of rectal cancer patients displaying variable responses to neoadjuvant radio chemotherapy, with the aim of studying whether disturbed $\mathrm{XIAP} / \mathrm{Smac}$ levels are indicative of resistance to neoadjuvant radio chemotherapy. Furthermore we examined matched pre and post treatment tissue to detect changes in XIAP or Smac expression induced by therapy. We also examined cIAP-1 and cIAP-2 expression in this tissue to examine whether these proteins may play a contributory role when XIAP/Smac balance is disturbed.

\section{Materials and methods Patient cohort}

Both biopsy and resection patient tissue was obtained from the Departments of Surgery, and Pathology, Beaumont Hospital, Dublin, Ireland. At colonoscopy or rigid sigmoidoscopy biopsy tumour and matched normal samples were collected. Biopsy tissue was collected in 21 male and 8 female patients (Table 1). The median age of patients was 65 , with ages ranging from 38 to 79 . To ensure consistent quality and tumour presence both normal and tumour tissues were evaluated by an experienced pathologist. Normal samples were obtained from a distant site in the rectal tract that was macroscopically unaffected and disease free. To be included in our analysis tumour samples must have contained over $50 \%$ tumour cells and normal samples must have been free of malignant cells. Following colonoscopy or rigid sigmoidoscopy patients underwent radio chemotherapy. The radio chemotherapy regimens consisted of radiotherapy (50.4 GY in 28 fractions) and 5FU (5-Fluorouracil), but some regimes varied slightly depending on tolerance of the patient. Once patients completed their course of treatment they underwent surgical resection. Post-treatment surgical resection tissue was obtained during surgery. Resection tissues were examined by a pathologist and graded based on regression of the tumour in response to therapy. Complete tumour regression (RCPath A) was achieved in 5 patients; partial tumour regression (RCPath $\mathrm{B}$ ) in 10 patients, and no marked tumour regression (RCPath C) was observed in 14 patients. A dedicated clinical research nurse reviewed medical records and gathered clinical information. Patients with a family history of colorectal cancer were excluded from analysis. Informed consent was obtained from all patients and Beaumont Hospital 
Table 1 Table of patient clinical characteristics

\begin{tabular}{|c|c|c|c|c|c|c|c|c|}
\hline \multirow[t]{2}{*}{ ID } & \multirow[t]{2}{*}{ Sex } & \multirow[t]{2}{*}{ Age } & \multirow{2}{*}{$\begin{array}{l}\text { Neoadjuvant } \\
\text { therapy }\end{array}$} & \multirow[t]{2}{*}{ RCPath } & \multirow{2}{*}{$\begin{array}{l}\text { Resection } \\
\text { tissue }\end{array}$} & \multicolumn{3}{|c|}{ Staging } \\
\hline & & & & & & $\mathrm{T}$ & $\mathrm{N}$ & M \\
\hline 1 & M & 73 & $50.4 / 28+5 f u$ & A & $x$ & 3 & 2 & 0 \\
\hline 2 & $\mathrm{~F}$ & 42 & $54 / 30+5 f u$ & A & $x$ & 2,3 & 1,2 & 0 \\
\hline 3 & $\mathrm{~F}$ & 38 & $50.4 / 28+5 f u$ & $A$ & $x$ & 3 & 1 & $x$ \\
\hline 4 & M & 75 & $54 / 31+5 f u$ & A & $x$ & 3 & 2 & $x$ \\
\hline 5 & M & 51 & $50.4 / 28+5 f u$ & A & $x$ & 3 & 2 & 0 \\
\hline 6 & M & 66 & $50.4 / 28+5 f u$ & B & $\sqrt{ }$ & 3 & 0,1 & 0 \\
\hline 7 & M & 57 & $50.4 / 28+5 f u$ & B & $\sqrt{ }$ & 3 & 1,2 & 0 \\
\hline 8 & $\mathrm{~F}$ & 72 & $50.4 / 28+5 f u$ & B & $x$ & 3 & 2 & 0 \\
\hline 9 & M & 65 & $50.4 / 28+5 f u$ & B & $x$ & 3 & $x$ & $x$ \\
\hline 10 & M & 65 & $50.4 / 28+5 f u$ & B & $x$ & 3 & 2 & $x$ \\
\hline 11 & $\mathrm{~F}$ & 64 & $42.2 / 20+5 f u$ & B & $x$ & 2 & 0 & 0 \\
\hline 12 & $\mathrm{~F}$ & 60 & $50.4 / 28+5 f u$ & B & $x$ & 2 & 0 & $x$ \\
\hline 13 & M & 66 & $50.4 / 28+5 f u$ & B & $x$ & 3 & 2 & 0 \\
\hline 14 & M & 67 & $50.4 / 28+5 f u$ & B & $x$ & 3 & 1 & 0 \\
\hline 15 & M & 67 & $54 / 30+5 f u$ & B & $x$ & 3 & 1 & $x$ \\
\hline 16 & $\mathrm{~F}$ & 55 & $55.4 / 30+5 f u$ & C & $\sqrt{ }$ & 3 & 2 & 0 \\
\hline 17 & $\mathrm{~F}$ & 75 & $45 / 25+5 f u / R P$ & C & $x$ & 3 & 0 & 0 \\
\hline 18 & M & 70 & $50.4 / 28+5 f u$ & C & $\sqrt{ }$ & 3 & 2 & 0 \\
\hline 19 & M & 79 & $50.4 / 28+5 f u$ & C & $x$ & 3 & 2 & 0 \\
\hline 20 & M & 76 & $43.2 / 25+5 f u$ & C & $\sqrt{ }$ & 3 & 2 & 0 \\
\hline 21 & M & 48 & $50.4 / 28+5 f u$ & C & $x$ & 3 & 0 & 0 \\
\hline 22 & M & 44 & $50.4 / 30+5 f u$ & C & $\sqrt{ }$ & 3 & 2 & 0 \\
\hline 23 & M & 47 & $50.4 / 28+5 f u$ & C & $\sqrt{ }$ & 3 & 2 & $x$ \\
\hline 24 & M & 74 & $50.4 / 28+5 f u$ & C & $\sqrt{ }$ & 3 & 2 & $x$ \\
\hline 25 & M & 45 & $50.4 / 28+5 f u$ & C & $x$ & 3 & 2 & $x$ \\
\hline 26 & M & 62 & $50.4 / 30+5 f u$ & $C$ & $x$ & 3 & 1 & $x$ \\
\hline 27 & M & 64 & $50.4 / 28+5 f u$ & C & $x$ & 3 & 1 & $x$ \\
\hline 28 & $\mathrm{~F}$ & 41 & $50.4 / 28+5 f u$ & C & $x$ & 3 & 1 & $x$ \\
\hline 29 & M & 71 & $50.4 / 28+5 f u$ & C & $x$ & 3 & 2 & $x$ \\
\hline
\end{tabular}

Ethics Committee granted ethical approval for the work.

\section{Protein extraction and quantification}

All tissue samples were lysed in $400 \mathrm{~mL}$ ice-cold buffer containing $50 \mathrm{mmol} / \mathrm{L}$ HEPES (pH 7.5), $150 \mathrm{mmol} / \mathrm{L}$ $\mathrm{NaCl}, 5 \mathrm{mmol} / \mathrm{L} \mathrm{Na}$-EDTA and protease inhibitor (Sigma). Samples were maintained on ice and homogenised using the Ultra-Turrax T25 Basic Homogeniser using $30 \mathrm{~s}$ pulses to break down the tissue. Protein concentrations were determined as follows, using the standard Pierce Micro-BCA Protein Assay (Pierce, Northumberland, UK): For calibration, a standard curve ranging from 0-12 $\mu \mathrm{g}$ was set up using a bovine serum albumin standard. Samples and standards were incubated at $37^{\circ} \mathrm{C}$ for $30 \mathrm{~min}$ and then absorbance was measured at $560 \mathrm{~nm}$. The average absorbances were calculated for each sample and standard. The slope of the BSA standard curve was used to calculate the protein concentration of each sample. Protein samples were prepared with SDS loading buffer (100 nM TRIS-Cl pH 6.8, 4 \% SDS, $0.2 \%$ bromophenol blue, $20 \%$ glycerol) and denatured at $95{ }^{\circ} \mathrm{C}$ for $10 \mathrm{~min}$. An equal amount of $20 \mu \mathrm{g}$ protein was loaded onto each lane of 10-15\% SDS-polyacrylamide gels. Gels were run at a voltage of $80 \mathrm{~V}$ until the proteins had migrated through the stacking gel, and then at $120 \mathrm{~V}$ to drive the proteins through the resolving gel. The running buffer was composed of $25 \mathrm{mM}$ Tris-Cl, $\mathrm{pH}$ 8.3, $250 \mathrm{mM}$ glycine and $0.1 \%$ SDS. Once the proteins had migrated through the gel the current was stopped and gels were transferred to Nitrocellulose membranes. Gels were blotted to nitrocellulose membranes (Protean BA 83; $2 \mu \mathrm{m}$; Schleicher \& Schuell) in transfer buffer (25 mM Tris, $192 \mathrm{mM}$ glycine, $20 \%$ methanol (v/v), and $0.01 \%$ SDS). Gels were transferred at $18 \mathrm{~V}$ for $60 \mathrm{~min}$. The nitrocellulose membranes were then blocked with $5 \%$ nonfat dry milk in TBST (15 mM Tris- $\mathrm{HCl}, \mathrm{pH} 7.5,200 \mathrm{mM} \mathrm{NaCl}$, and $0.1 \%$ Tween 20) at room temperature for $1 \mathrm{~h}$. Membranes were then incubated with the primary antibodies overnight at $4{ }^{\circ} \mathrm{C}$. The following primary antibodies were used: XIAP (1:1000; Mouse monoclonal, 610762, BD transduction laboratories, USA), Smac (1:1000; Rabbit polyclonal, AF789 R\&D Systems, USA), cIAP1 (1:1000; Rabbit polyclonal, \#4952, CST), cIAP2 (1:1000; Rabbit monoclonal, \#3130, CST) and b-actin (1:5000; Sigma). Secondary antibodies were horseradish peroxidase (HRP)-conjugated and were used at a dilution of 1:10,000 (Millipore), and membranes were incubated for $1 \mathrm{~h}$ at room temperature. Blots were developed using the enhanced chemiluminescence detection reagent (Millipore, Ireland). Chemiluminescence was detected at 12-bit dynamic range using a Fuji LAS 4000 CCD system (Fujifilm UK Ltd., Bedfordshire, UK). Densitometry was then performed. The intensity of each band was calculated using Image J software. The intensity of the loading control was deducted from the intensity of the band of interest to eliminate any differences in protein levels owing to uneven loading, so that each sample was normalized and could be compared. Each sample was normalised to the intensity of its corresponding $\beta$-Actin band.

\section{Statistics}

Statistical analyses were performed in Matlab (MathWorks, Natick, MA, USA), and SPSS (IBM, Armonk, NY, USA). Data are given as means \pm s.e.m. For statistical comparison Student's $t$ test or ANOVA and subsequent Tukey test were used for normal distributed data. $P$ values $\leq 0.05$ were considered statistically significant. 


\section{Results}

XIAP level increases in accordance with tissue resistance to radio chemotherapy in tumour tissue, but not normal tissue

Pre-treatment biopsy tumour and matched normal tissue samples were obtained at colonoscopy or rigid sigmoidoscopy (Fig. 1) from 29 rectal cancer patients. Using quantitative Western blotting, the expression levels of XIAP and Smac in tumour and matched normal biopsy tissue were determined in all 29 patients. Representative Western blot images for 6 patients (3 RCPath A, 2 RCPath B and 1 RCPath C) are shown in Fig. 2a. Beta Actin was used as a loading control. HeLa cells were used as a standard as previously described [28] (Fig. 2a). Clinical data was then examined and protein levels were correlated to RCPath grade (Table 1).

When we examined expression levels in all 29 patients we see that as tumour tissue became more radio chemotherapy resistant, from RCPath A to RCPath C, XIAP levels significantly increased $(p=0.025)$ (Fig. 2b). Conversely Smac levels did not increase with RCPath grade (Fig. 2c). This indicated a shift in the expression of these two proteins as tissue becomes more resistant to therapy. While this pattern was very evident in tumour

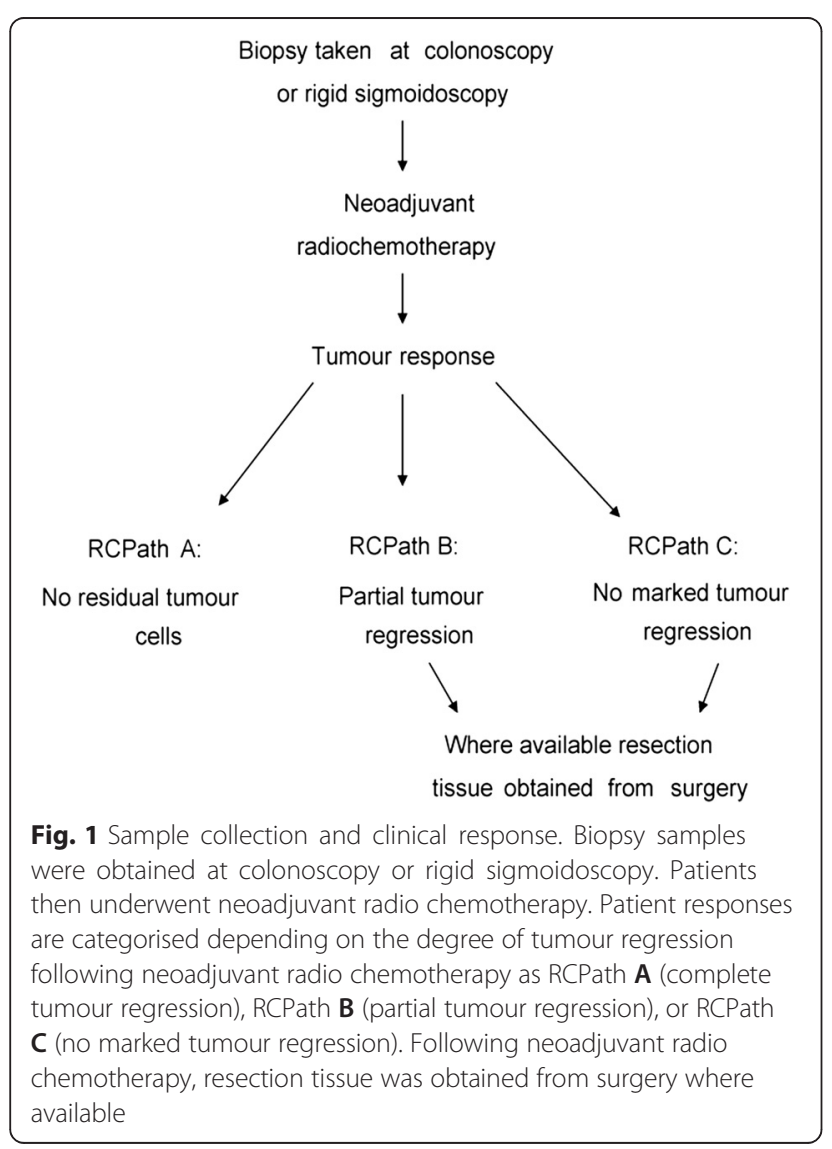

tissue, matched normal tissue did not follow this trend. In normal tissue both XIAP and Smac levels remained unchanged throughout the varying grades of radio chemotherapy resistance. In RCPath $\mathrm{C}$ patients XIAP protein levels were significantly raised in the tumour tissue compared to the matched normal tissue $(p=0.007)$. These data demonstrated the importance of the XIAP/ Smac balance and as XIAP levels increase and outweigh Smac levels, cells may be able to avoid apoptosis, via increased caspase inhibition, and thus become more resistant to therapy. Due to limited availability of biopsy tissue cIAP-1 and-2 were examined in only 14 biopsy tissues. Of these tissues 2 were RCPath A, 4 were RCPath $\mathrm{B}$ and 8 were RCPath $\mathrm{C}$. Consequently RCPath A and B were pooled and compared to RCPath C. Both cIAP-1 and- 2 protein levels were similar in all patients regardless of pathological response to neoadjuvant radio chemotherapy, suggesting that these proteins do not play a contributory role when the XIAP/Smac balance is disturbed (Fig. 2d, e). When we investigated whether XIAP level in biopsy tissue may have predictive power in selecting patients who respond to therapy, we found that XIAP levels could predict patients that responded to therapy with a sensitivity of $80 \%$ and a specificity of $88 \%$, outperforming TNM staging which could not predict patient response to therapy, with a sensitivity $20 \%$ and a specificity $100 \%$.

\section{XIAP expression increases in tumour tissue during radio chemotherapy}

Following neoadjuvant radio chemotherapy patients underwent surgery to remove any remaining tumour. In RCPath A patients, where a complete pathological response was achieved, no surgical resection tissue was available. In 8 RCPath B and C patients, where neoadjuvant radio chemotherapy induced only partial tumour regression, or no tumour regression respectively, surgical resection tissue was available post treatment (Fig. 1).

Using Western blotting we examined XIAP, Smac and cIAP-1 and-2 levels in these samples. XIAP levels were compared in pretreatment biopsy tissue and post treatment resection tissue. In tumour (Fig. 3a) XIAP expression increased significantly during the course of neoadjuvant radio chemotherapy $(p=0.004662)$. XIAP expression in matched normal tissue also increased but not significantly $(p=0.082984)$. Smac levels were also compared in pretreatment biopsy tissue and post treatment resection tissue (Fig. 4a, b). We saw no significant change in Smac expression in normal or tumour tissue pre and post treatment. This suggested that cancer cells that survive treatment responded by increasing their XIAP protein levels which may have resulted in radio chemotherapy resistance. Due to limited availability of 


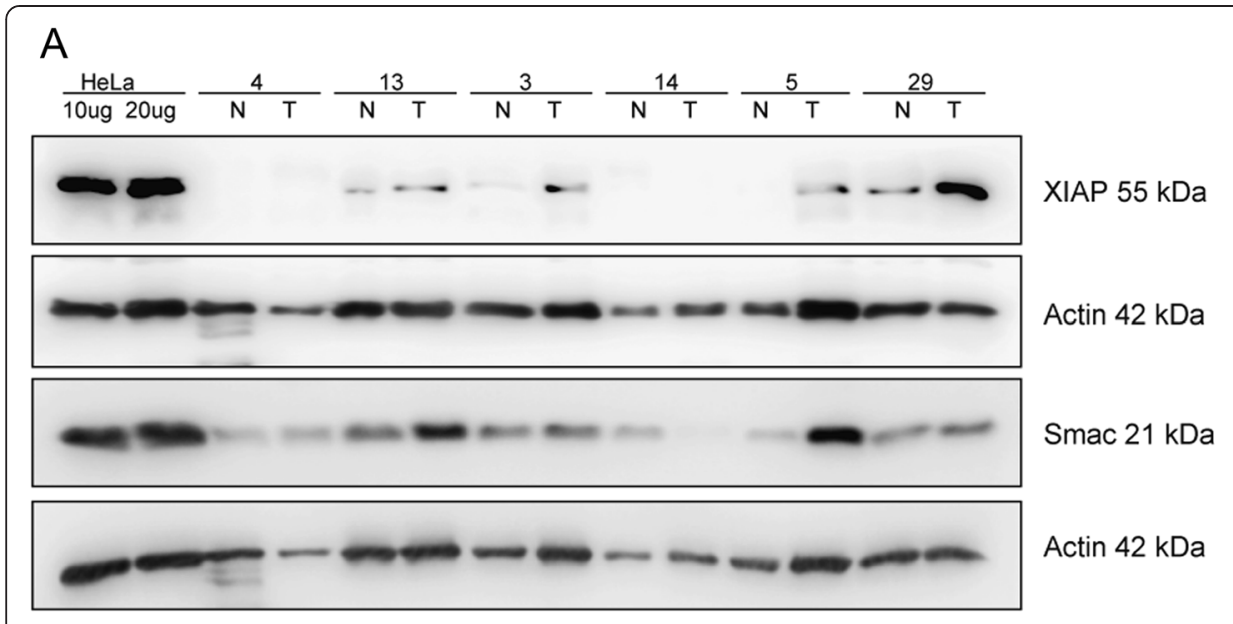

B XIAP protein level in pre treatment biopsy tissue

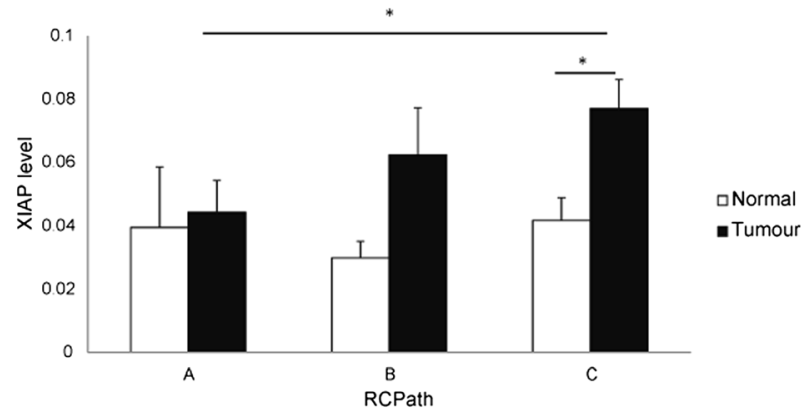

D

cIAP1 protein level in pre treatment biopsy tissue

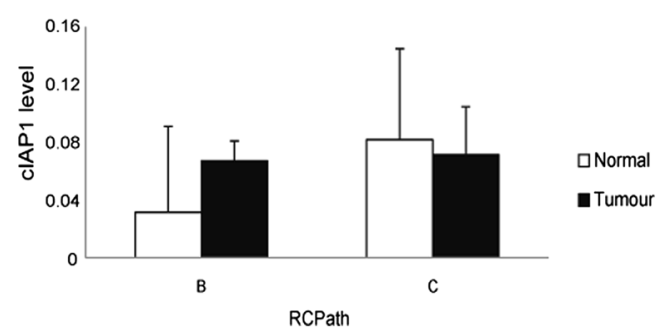

C Smac protein level in pre treatment biopsy tissue

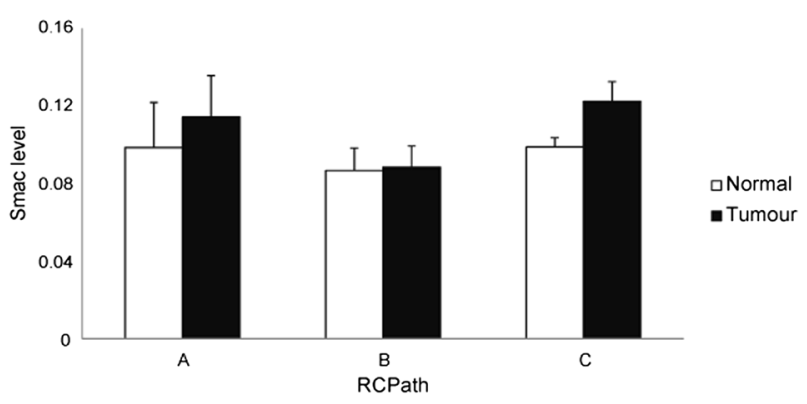

$\mathrm{E}$

cIAP2 protein level in pre treatment biopsy tissue

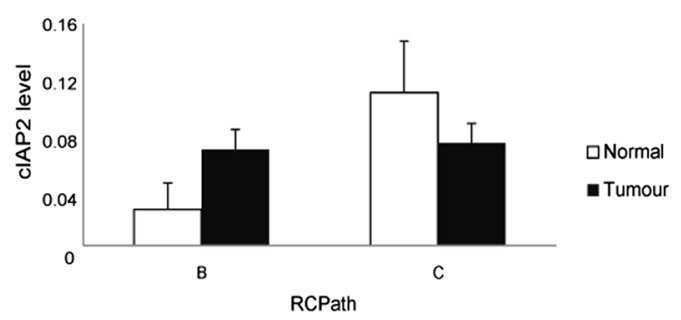

Fig. 2 Elevated XIAP protein levels prior to neoadjuvant radio chemotherapy indicate resistance to therapy in rectal cancer patients. Biopsy tissue samples of 29 rectal cancer patients were obtained at colonoscopy or rigid sigmoidoscopy. a Representative Western blot images for 6 patients (3 RCPath A, 2 RCPath B and 1 RCPath C) are shown in Fig. 2a. The expression levels of XIAP and Smac in tumour (T) and matched normal tissue (N) were determined by quantitative Western blotting. Beta Actin was used as a loading control. HeLa cells were used as a standard. b Protein expression levels for all 29 patients were then correlated with tumour regression grade. Levels of XIAP protein increased in accordance with radio chemotherapy resistance $(p=0.025)$ in tumour tissue. XIAP protein levels are also significantly raised in the tumour tissue compared to the matched normal tissue in RCPath C patients ( $p=0.007)$. c Smac protein was not differentially expressed in RCPath grades. Furthermore there was no significant difference in Smac expression in tumour and matched normal tissue. ( $\mathbf{d}, \mathbf{e})$ Expression levels of cIAP-1 (d) and clAP-2 (e) were also determined by quantitative Western blotting in 14 biopsy tissues and then correlated with tumour regression grade. Neither protein was differentially expressed in RCPath grades nor was there a significant difference in expression in tumour and matched normal tissue

tissue cIAP-1 and-2 was examined in tumour tissue only (Fig. 5a, b; cIAP-1 $n=6$; cIAP-2 $n=5$ ). Neither protein was affected by neoadjuvant radio chemotherapy, with no changes seen in expression levels in tumour tissue pre and post treatment.

\section{Discussion}

In rectal cancer patients routinely undergo neoadjuvant radio chemotherapy. Radio chemotherapy, and also radiotherapy alone, prior to surgery has been shown to reduce rates of local recurrence and improve disease free survival, 
A XIAP level in biopsy and resection Tumour tissue

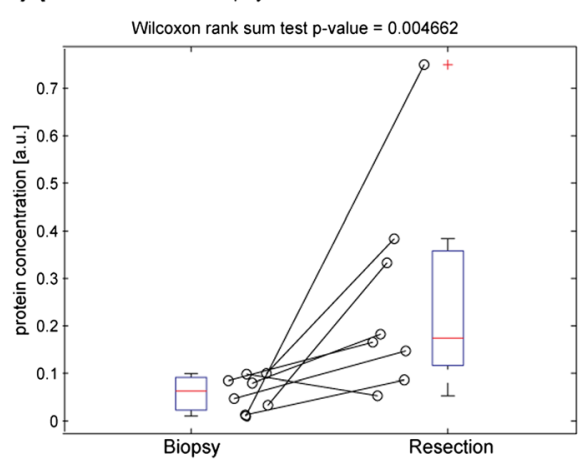

B XIAP level in biopsy and resection Normal tissue

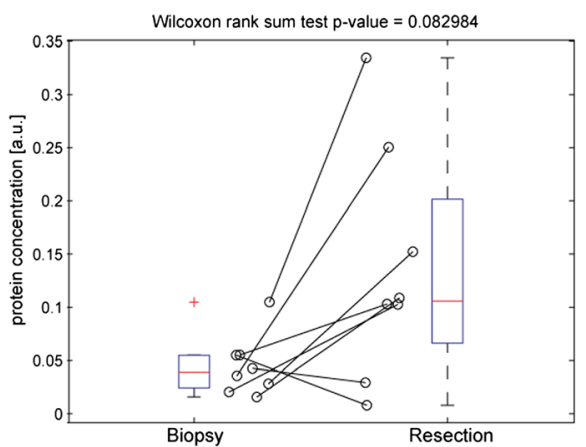

Fig. 3 XIAP expression increases in tumour tissue during radio chemotherapy. For 8 patients where pre-treatment biopsy and matched posttreatment surgical resection tissue was available the expression levels of XIAP in tumour and matched normal tissue were once again determined by quantitative Western blotting. a In individual patients, all but one patient had increased XIAP levels in resection tumour tissue, compared to matched tumour biopsy tissue, and XIAP expression was significantly increased in post treatment resection tumour tissue, when compared to pre-treatment biopsy tumour tissue $(p=0.004662)$. $\mathbf{b}$ In matched normal tissue 6 out of 8 patients had increased XIAP levels in resection tumour tissue, compared to matched tumour biopsy tissue. This trend was not significant

when compared to surgery alone [29, 30]. Patients who experience complete pathological response to neo-adjuvant radio chemotherapy experience low incidence of local recurrence and distant metastases [7, 8]. A response to neo-adjuvant radio chemotherapy of $95 \%$ or greater is associated with a good long term outcome for the patient [31]. Many patients respond poorly to neoadjuvant radio chemotherapy but the reason for this is currently not well understood $[9,10,32]$. We examined XIAP, cIAP-1, cIAP2 and Smac protein levels in a cohort of rectal cancer patients to examine whether more chemo resistant tissue displayed an altered protein expression. We found that XIAP levels in tumour tissue increased as chemo resistance grades progressed from $\mathrm{RCPath} A$, through RCPath $B$, to RCPath $C$, suggesting XIAP may play a critical role in resistance to neoadjuvant radio chemotherapy. Furthermore our study highlights the potential of XIAP as a marker of response to neoadjuvant radio chemotherapy.

XIAP expression has previously been shown to be an important factor in cancer progression. In colorectal cancer cells high XIAP mRNA levels have been shown to be indicative of tumour differentiation, invasion and progression [33]. As well as contributing to disease progression XIAP was also reported to contribute to chemotherapy resistance [34]. In a cohort of 90 ovarian

\section{A Smac level in biopsy and resection Tumour tissue}

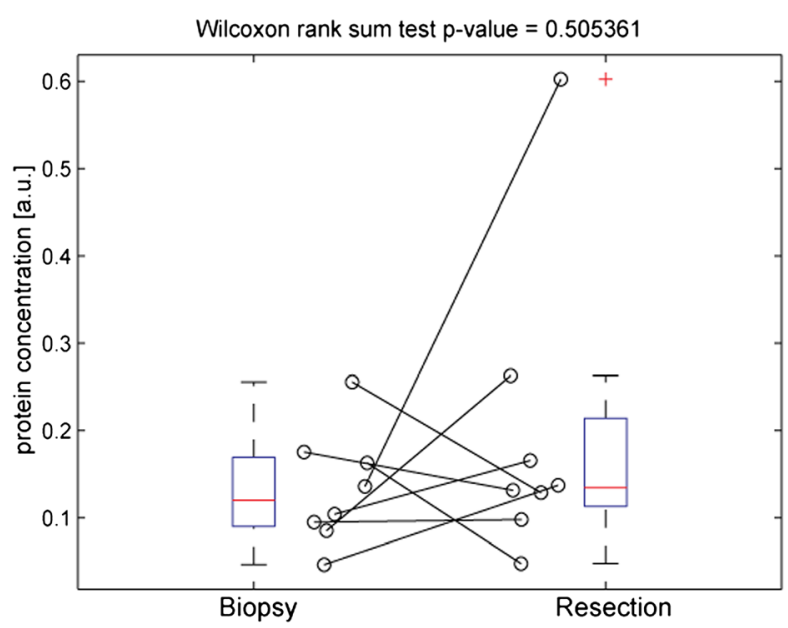

\section{B Smac level in biopsy and resection Normal tissue}

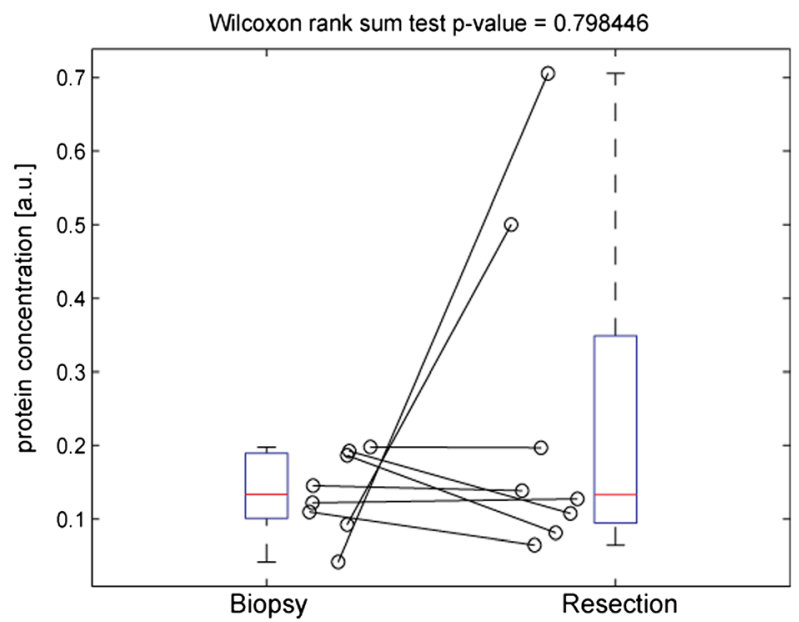

Fig. 4 Smac expression does not change in normal and tumour tissue during radio chemotherapy. Smac expression levels in tumour and matched normal tissue were once again determined by quantitative Western blotting in pre-treatment biopsy and matched post-treatment surgical resection tissue, which was available for 8 patients. Smac expression was not significantly increased in post treatment resection (a) tumour and (b) normal tissue, when compared to pre-treatment biopsy tumour and normal tissue 


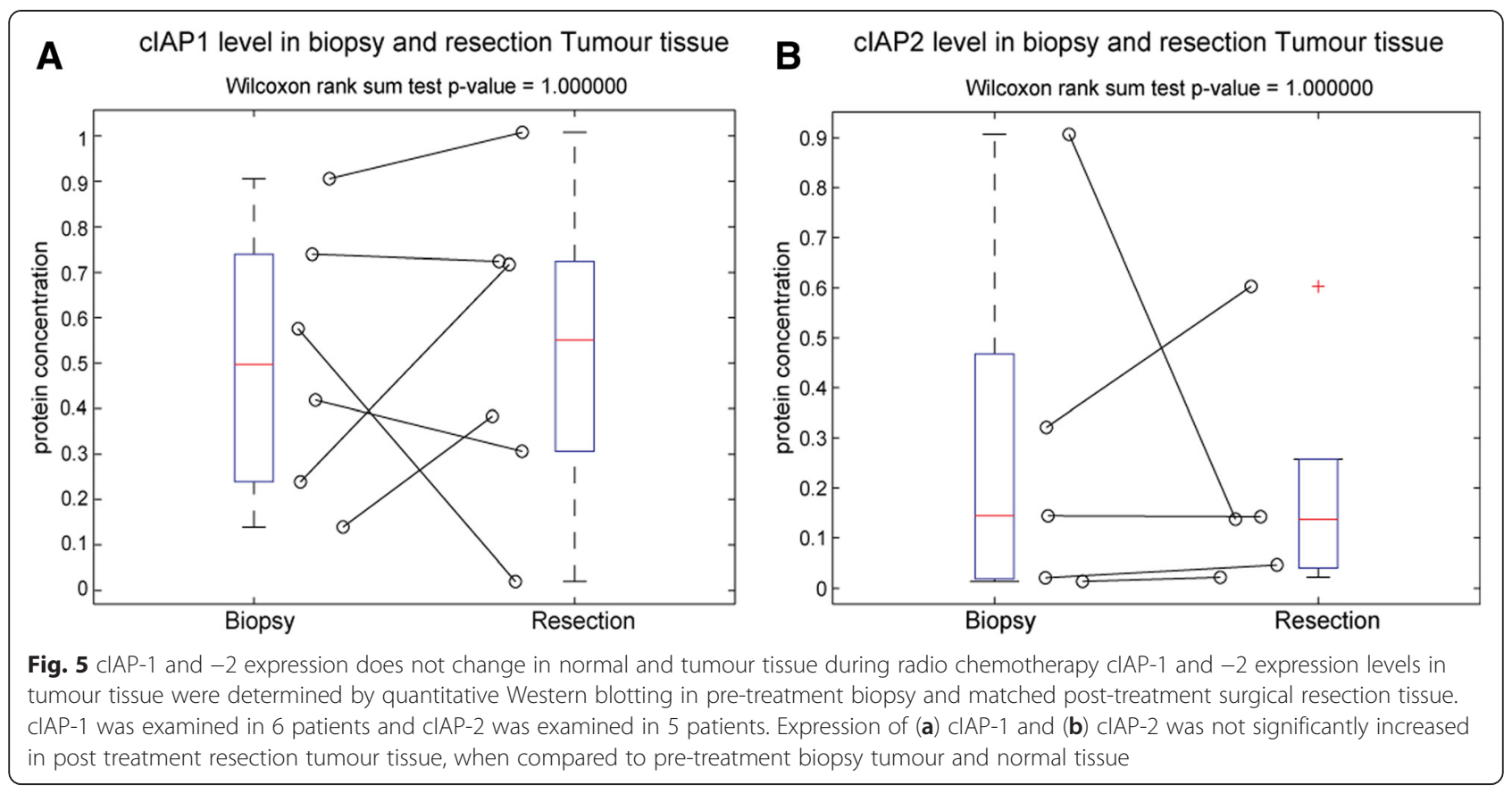

clear cell carcinoma patients high XIAP expression was found to correlate with lower chemotherapy response rates and also a worse progression free and overall survival for patients [19]. High levels of XIAP in lung cancer cells resulted in cisplatin mediated apoptosis resistance, and this apoptosis could be restored once XIAP was silenced [20]. Similar trends were reported in colorectal and breast cancer cell lines, where ceramide was found to effectively sensitise cells to apoptosis and also suppress tumour progression, via targeting of XIAP [18]. These studies highlight the importance of XIAP in cancer progression and demonstrate a crucial role for XIAP in triggering resistance to chemotherapeutic drugs.

Our work demonstrated that while XIAP expression increased as chemo resistance grades progressed from RCPath A, through RCPath B, to RCPath C, Smac levels remained constant. Smac is XIAP's main antagonist, blocking XIAP's caspase inhibitory function and allowing apoptosis to proceed. CIAP-1 and cIAP-2 are other inhibitor of apoptosis proteins that Smac binds to. As the XIAP/Smac balance shifts we saw no differential regulation of these proteins indicating that they play no contributory role when XIAP/Smac balance is disturbed. In many cancer cells as XIAP levels increase Smac levels do not mirror this pushing the cells into a more antiapoptotic state. Findings similar to ours were previously reported in renal cell carcinomas (RCC). Yan et al. investigated XIAP and Smac expression in RCC, and found that Smac levels remained constant regardless of tumor progression, despite XIAP expression increasing significantly from early to advanced tumor stages. This disturbed XIAP/Smac balance may contribute to apoptotic resistance in RCC [25]. Another scenario where disruption of this protein balance was found to contribute to disease progression is Hodgkins disease. Hodgkins and Reed Sternberg cells and Hodgkin lymphoma derived B cells involved in this disease express high levels of XIAP. When the XIAP/Smac balance was restored in these cells they become re-sensitised to apoptosis [26].

As XIAP levels rise and the XIAP/Smac balance shifts Smac levels may not be sufficient to overcome XIAP's anti-apoptotic action. Restoration of this balance may push cells back towards apoptosis and hence synthetic Smac peptides and small molecule Smac mimetics are being examined as novel cancer therapeutics. In combination with chemotherapy and radiotherapy these compounds have been reported to sensitise resistant cancer cells to apoptosis via modulation of both the apoptotic and NF- $\mathrm{kB}$ pathways [35-37]. The importance of the Smac/XIAP ratio in treatment responses to Smac mimetics was highlighted in childhood acute lymphoblastic leukaemia where XIAP is upregulated. Antagonism of XIAP, via a Smac mimetic, led to a significant increase in apoptosis in this setting [16]. Combined Smac mimetics and TRAIL treatment also reduced metastatic behaviour and cell migration [38]. Recent reports have highlighted that Smac mimetics can also trigger necroptosis as an alternative form of cell death to overcome apoptosis resistance in acute myeloid leukaemia cells [39]. Our data suggests that in the rectal cancer setting those patients who were unresponsive to traditional therapies and displayed high XIAP levels may benefit from Smac mimetic treatment to re-establish the XIAP/ Smac balance, and in turn re-sensitise cells to therapy. 
As well as increasing expression in radio chemotherapy resistant cells, we found that XIAP expression was also up regulated by neoadjuvant radio chemotherapy. Levels of XIAP in both normal and tumour tissue were significantly increased in post treatment surgical resection tissue, when compared to pretreatment biopsy tissue. Smac levels did not increase in response to therapy in either normal or tumour tissue, which once again contributes to a disruption of XIAP/Smac expression in cells. Recently it has been reported that chemotherapy induced enhanced XIAP expression, partially mediated through PI3K/Akt signalling, resulting in chemo resistance in breast cancer cells [40]. These data suggest that following treatment sensitive cells are killed off but a cohort of more resistant cells with high levels of XIAP remain. This has significant implications for further adjuvant treatment, as this more resistant population may continue to proliferate and be refractory to traditional therapies, highlighting another scenario where Smac mimetics would be an attractive alternative treatment regime.

\section{Conclusions}

Our work highlights XIAP's central role in resistance to neoadjuvant radio chemotherapy, leading to reduced therapy success. The use of Smac mimetics in this setting may restore the XIAP/Smac balance, in turn restoring apoptosis and leading to improved patient outcomes. Furthermore XIAP levels increase in response to neoadjuvant radio chemotherapy. Up regulation of this protein may lead to a more resistant phenotype in the adjuvant treatment setting, and patients defined by this XIAP over expression may benefit from alternative adjuvant treatment regimes, such as Smac mimetics.

\section{Abbreviations \\ XIAP: X-linked inhibitor of apoptosis protein; Smac: Second mitochondria- derived activator of caspases; TRAIL: Tumor necrosis factor-related apoptosis- inducing ligand; HRP: Horseradish peroxidase; T N M: Tumour, node, and metastasis; RCC: Renal cell carcinomas; 5fu: 5-Fluorouracil; RCPath: Royal College of Pathologists.}

\section{Competing interests}

The authors declare that they have no competing interests.

\begin{abstract}
Authors' contributions
Conception and design: LF, JHMP. Acquisition and processing of data: LF, DAM, JD, EWK, JF, OB, JK. Analysis and interpretation of data: LF, AUL, EWK, $O B, J K, D A M, J D, J H M P$. Writing, review, and/or revision of the manuscript: LF, DAM, JHMP. Administrative, technical, or material support: OB, EWK, DAM, JD, JHMP. Study supervision: DAM, JHMP. All authors read and approved the final manuscript.
\end{abstract}

\footnotetext{
Author details

${ }^{1}$ Department of Physiology and Medical Physics, Royal College of Surgeons in Ireland, 123 St. Stephen's Green, Dublin 2, Ireland. ${ }^{2}$ Centre for Systems Medicine, Royal College of Surgeons in Ireland, 123 St. Stephen's Green, Dublin 2, Ireland. ${ }^{3}$ Departments of Pathology, Beaumont Hospital, Dublin 9, Ireland. ${ }^{4}$ Departments of Surgery, Beaumont Hospital, Dublin 9, Ireland.
}

Received: 4 March 2015 Accepted: 5 June 2015

Published online: 13 June 2015

\section{References}

1. Thompson CB. Apoptosis in the pathogenesis and treatment of disease. Science. 1995;267(5203):1456-62. doi:10.1126/science.7878464.

2. Brown JM, Attardi LD. The role of apoptosis in cancer development and treatment response. Nat Rev Cancer. 2005;5(3):231-7. doi:10.1038/nrc1560.

3. Gougeon ML, Poccia F, Boullier S. Human gamma delta T lymphocytes in HIV disease: effector functions and control by natural killer cell receptors. Springer Semin Immunopathol. 2000;22(3):251-63.

4. Plati J, Bucur O, Khosravi-Far R. Dysregulation of apoptotic signaling in cancer: molecular mechanisms and therapeutic opportunities. J Cell Biochem. 2008;104(4):1124-49. doi:10.1002/jcb.21707.

5. Medich D, McGinty J, Parda D, Karlovits S, Davis C, Caushaj P, et al. Preoperative chemoradiotherapy and radical surgery for locally advanced distal rectal adenocarcinoma. Dis Colon Rectum. 2001;44(8):1123-8. doi:10.1007/bf02234632.

6. Williams GT, Quirke P, Shepherd NA. Dataset for colorectal cancer (2nd edition). In: Pathologists TRCo, editor. 2007.

7. Janjan NA, Khoo VS, Abbruzzese J, Pazdur R, Dubrow R, Cleary KR, et al. Tumor downstaging and sphincter preservation with preoperative chemoradiation in locally advanced rectal cancer: the M. D. Anderson Cancer Center experience. Int J Radiat Oncol Biol Phys. 1999;44(5):1027-38. doi:10.1016/S0360-3016(99)00099-1.

8. Maas M, Nelemans PJ, Valentini V, Das P, Rödel C, Kuo L-J, et al. Long-term outcome in patients with a pathological complete response after chemoradiation for rectal cancer: a pooled analysis of individual patient data. Lancet Oncol. 2010;11(9):835-44. doi:10.1016/S1470-2045(10)70172-8.

9. Lu J-Y, Xiao Y, Qiu H-Z, Wu B, Lin G-L, Xu L, et al. Clinical outcome of neoadjuvant chemoradiation therapy with oxaliplatin and capecitabine or 5-fluorouracil for locally advanced rectal cancer. J Surg Oncol. 2013;108(4):213-9. doi:10.1002/jso.23394.

10. Janjan NA, Crane C, Feig BW, Cleary K, Dubrow R, Curley S, et al. Improved overall survival among responders to preoperative chemoradiation for locally advanced rectal cancer. Am J Clin Oncol. 2001;24(2):107-12.

11. Duckett CS, Nava VE, Gedrich RW, Clem RJ, Van Dongen JL, Gilfillan MC, et al. A conserved family of cellular genes related to the baculovirus iap gene and encoding apoptosis inhibitors. EMBO J. 1996;15(11):2685-94.

12. Liston P, Roy N, Tamai K, Lefebvre C, Baird S, Cherton-Horvat G, et al. Suppression of apoptosis in mammalian cells by NAIP and a related family of IAP genes. Nature. 1996;379(6563):349-53. doi:10.1038/379349a0.

13. Rothe M, Pan MG, Henzel WJ, Ayres TM, Goeddel DV. The TNFR2-TRAF signaling complex contains two novel proteins related to baculoviral inhibitor of apoptosis proteins. Cell. 1995;83(7):1243-52.

14. Seligson DB, Hongo F, Huerta-Yepez S, Mizutani Y, Miki T, Yu H, et al. Expression of $X$-Linked Inhibitor of Apoptosis Protein Is a Strong Predictor of Human Prostate Cancer Recurrence. Clin Cancer Res. 2007;13(20):6056-63. doi:10.1158/1078-0432.ccr-07-0960.

15. Tamm I, Kornblau SM, Segall H, Krajewski S, Welsh K, Kitada S, et al. Expression and Prognostic Significance of IAP-Family Genes in Human Cancers and Myeloid Leukemias. Clin Cancer Res. 2000;6(5):1796-803.

16. Hundsdoerfer P, Dietrich I, Schmelz K, Eckert C, Henze G. XIAP expression is post-transcriptionally upregulated in childhood ALL and is associated with glucocorticoid response in T-cell ALL. Pediatr Blood Cancer. 2010;55(2):260-6. doi:10.1002/pbc.22541.

17. Zhou S, Ye W, Shao Q, Qi Y, Zhang M, Liang J. Prognostic significance of XIAP and NF-KB expression in esophageal carcinoma with postoperative radiotherapy. World J Surg Oncol. 2013;11:288. doi:10.1186/1477-7819-11-288.

18. Paschall AV, Zimmerman MA, Torres CM, Yang D, Chen MR, Li X, et al. Ceramide targets XIAP and CIAP1 to sensitize metastatic colon and breast cancer cells to apoptosis induction to suppress tumor progression. BMC Cancer. 2014;14:24. doi:10.1186/1471-2407-14-24.

19. Miyamoto M, Takano M, Iwaya K, Shinomiya N, Kato M, Aoyama T, et al. X-chromosome-linked inhibitor of apoptosis as a key factor for chemoresistance in clear cell carcinoma of the ovary. Br J Cancer. 2014;110(12):2881-6. doi:10.1038/bjc.2014.255.

20. Cheng YJ, Jiang HS, Hsu SL, Lin LC, Wu CL, Ghanta VK, et al. XIAP-mediated protection of $\mathrm{H} 460$ lung cancer cells against cisplatin. Eur J Pharmacol. 2010;627(1-3):75-84. doi:10.1016/j.ejphar.2009.11.003. 
21. Huerta S, Gao X, Saha D. Mechanisms of resistance to ionizing radiation in rectal cancer. Expert Rev Mol Diagn. 2009;9(5):469-80. doi:10.1586/erm.09.26.

22. Varfolomeev E, Blankenship JW, Wayson SM, Fedorova AV, Kayagaki N, Garg $P$, et al. IAP antagonists induce autoubiquitination of c-IAPs, NF-kappaB activation, and TNFalpha-dependent apoptosis. Cell. 2007;131(4):669-81.

23. Vince JE, Wong WW, Khan N, Feltham R, Chau D, Ahmed AU, et al. IAP antagonists target CIAP1 to induce TNFalpha-dependent apoptosis. Cell. 2007;131(4):682-93.

24. Hu S, Yang $X$. Cellular inhibitor of apoptosis 1 and 2 are ubiquitin ligases for the apoptosis inducer Smac/DIABLO. J Biol Chem. 2003;278(12):10055-60.

25. Yan Y, Mahotka C, Heikaus S, Shibata T, Wethkamp N, Liebmann J, et al. Disturbed balance of expression between XIAP and Smac/DIABLO during tumour progression in renal cell carcinomas. Br J Cancer. 2004;91(7):1349-57.

26. Kashkar H, Haefs C, Shin H, Hamilton-Dutoit SJ, Salvesen GS, Kronke M, et al. XIAP-mediated caspase inhibition in Hodgkin's lymphoma-derived B cells. J Exp Med. 2003;198(2):341-7. doi:10.1084/jem.20021279jem.20021279.

27. Dai $Y$, Lawrence $T, X u L$. Overcoming cancer therapy resistance by targeting inhibitors of apoptosis proteins and nuclear factor-kappa B. Am J Transl Res. 2009;1(1):4-18.

28. Hector S, Rehm M, Schmid J, Kehoe J, McCawley N, Dicker P, et al. Clinical application of a systems model of apoptosis execution for the prediction of colorectal cancer therapy responses and personalisation of therapy. Gut. 2012;61(5):725-33. doi:10.1136/gutjnl-2011-300433.

29. Rödel C, Martus P, Papadoupolos T, Füzesi L, Klimpfinger M, Fietkau R, et al. Prognostic Significance of Tumor Regression After Preoperative Chemoradiotherapy for Rectal Cancer. J Clin Oncol. 2005;23(34):8688-96. doi:10.1200/jco.2005.02.1329.

30. van Gijn W, Marijnen CA, Nagtegaal ID, Kranenbarg EM, Putter H, Wiggers $\mathrm{T}$, et al. Preoperative radiotherapy combined with total mesorectal excision for resectable rectal cancer: 12-year follow-up of the multicentre, randomised controlled TME trial. Lancet Oncol. 2011;12(6):575-82. doi:10.1016/S1470-2045(11)70097-3.

31. Ruo L, Tickoo S, Klimstra DS, Minsky BD, Saltz L, Mazumdar M, et al. Long-term prognostic significance of extent of rectal cancer response to preoperative radiation and chemotherapy. Ann Surg. 2002;236(1):75-81.

32. Huerta S, Hrom J, Gao X, Saha D, Anthony T, Reinhart H, et al. Tissue microarray constructs to predict a response to chemoradiation in rectal cancer. Dig Liver Dis. 2010;42(10):679-84. doi:10.1016/j.dld.2010.02.003.

33. Xiang $G$, Wen $X$, Wang $H$, Chen $K$, Liu $H$. Expression of $X$-linked inhibitor of apoptosis protein in human colorectal cancer and its correlation with prognosis. J Surg Oncol. 2009;100(8):708-12. doi:10.1002/jso.21408.

34. Castells M, Milhas D, Gandy C, Thibault B, Rafii A, Delord JP, et al. Microenvironment mesenchymal cells protect ovarian cancer cell lines from apoptosis by inhibiting XIAP inactivation. Cell Death Dis. 2013;4:e887. doi:10.1038/cddis.2013.384.

35. Dai Y, Liu M, Tang W, Li Y, Lian J, Lawrence TS, et al. A Smac-mimetic sensitizes prostate cancer cells to TRAlL-induced apoptosis via modulating both IAPs and NF-kappaB. BMC Cancer. 2009;9:392.

36. Huang Y, Park YC, Rich RL, Segal D, Myszka DG, Wu H. Structural Basis of Caspase Inhibition by XIAP: Differential Roles of the Linker versus the BIR Domain. Cell. 2001;104(5):781.

37. Huerta S, Gao X, Livingston EH, Kapur P, Sun H, Anthony T. In vitro and in vivo radiosensitization of colorectal cancer HT-29 cells by the smac mimetic JP-1201. Surgery. 2010;148(2):346-53. doi:10.1016/j.surg.2010.05.006.

38. Fingas CD, Blechacz BR, Smoot RL, Guicciardi ME, Mott J, Bronk SF, et al. A smac mimetic reduces TNF related apoptosis inducing ligand (TRAIL)induced invasion and metastasis of cholangiocarcinoma cells. Hepatology. 2010;52(2):550-61. doi:10.1002/hep.23729.

39. Chromik J, Safferthal C, Serve H, Fulda S. Smac mimetic primes apoptosisresistant acute myeloid leukaemia cells for cytarabine-induced cell death by triggering necroptosis. Cancer Letters. 2014;344(1):101-9. doi:10.1016/j.canlet.2013.10.018.

40. Ghebeh H, Al-Khaldi S, Olabi S, Al-Dhfyan A, Al-Mohanna F, Barnawi R, et al. Fascin is involved in the chemotherapeutic resistance of breast cancer cells predominantly via the PI3K/Akt pathway. Br J Cancer. 2014;111(8):1552-61. doi:10.1038/bjc.2014.453.

\section{Submit your next manuscript to BioMed Central and take full advantage of:}

- Convenient online submission

- Thorough peer review

- No space constraints or color figure charges

- Immediate publication on acceptance

- Inclusion in PubMed, CAS, Scopus and Google Scholar

- Research which is freely available for redistribution

Submit your manuscript at www.biomedcentral.com/submit 